$\frac{51}{2} / 948 S(2)$ PARTICLE TRANSPORT DUE TO MAGNETIC FLUCTUATIONS

M.R. Stoneking, S.A. Hokin, S.C. Prager,

G. Fiksel, H. Ji, D.J. Denhartog 


\section{NOTICE}

This report was prepared as an account of work sponsored by an agency of the United States Government. Neither the United States nor any agency thereof, nor any of their employees, makes any warranty, expressed or implied, or assumes any legal liability or responsibility for any third party's use or the results of such use of any information, apparatus, product or process disclosed in this report, or represents that its use by such third party would not infringe privately owned rights.

Printed in the United States of America

Available from

National Technical Information Service

U.S. Department of Commerce

5285 Port Royal Road

Springfield, VA 22161

NTIS Price codes

Printed copy:

$\mathrm{A} 02$

Microfiche copy: A01 


\title{
Particle Transport Due to Magnetic Fluctuations
}

\author{
M. R. Stoneking, S. A. Hokin, S. C. Prager, G. Fiksel, H. Ji, and D. J. Den Hartog \\ Department of Physics \\ University of Wisconsin, Madison, WI 53706
}

Electron current fluctuations are measured with an electrostatic energy anaijzer at the edge of the MST reversed-field pinch plasma. The radial flux of fast electrons $\left(E>T_{e}\right)$ due to parallel streaming along a fluctuating magnetic field is determined locally by measuring the correlated product $\left\langle\tilde{J}_{e} \tilde{B}_{r}\right\rangle$. Particle transport is small just inside the last closed flux surface $\left(\Gamma_{e, m a g}<0.1 \Gamma_{\theta, \text { totall), but can }}\right.$ account for all observed particle losses inside r/a=0.8. Electron diffusion is found to increase with parallel velocity, as expected for diffusion in a region of field stochasticity.

Plasma transport across a confining magnetic field continues to be a central problem in the field of plasma physics, engendering numerous experimental, theoretical and computational studies. The mechanisms responsible for fluctuation-induced transport are broadly classified as either electrostatic or magnetic in origin. For example, radial electron particle flux can be described by 1

$$
\Gamma_{r, \theta}=\frac{\left\langle\tilde{n} \tilde{E}_{\perp}\right\rangle}{B}-\frac{\left\langle\tilde{J}_{1, \theta} \tilde{B}_{r}\right\rangle}{\Theta B},
$$

where the symbol $\langle\tilde{a} \tilde{b}\rangle$ indicates the flux surface averaged product of the fluctuating quantities (density $\tilde{n}$, perpendicular electric field $\tilde{E}_{\perp}$, parallel electron current $\tilde{J}_{1, e}$, and radial magnetic field $\tilde{B}_{r}$ ), and $B$ is the magnitude of the confining field. The first term in Eq. 1 represents the electrostatic contribution to the flux and results from the fluctuating $\tilde{E} \times B$ guiding center drift. Fluctuations in 
the plasma density which are correlated with local electric field fluctuations result in outward electrostatic particle transport if the relative phase of the fluctuations is not $\pi / 2$ radians. The second term describes the magnetic transport due to fluctuations in the electron current which are correlated and in phase with fluctuations in $\mathrm{Br}$. Measurements of the local electrostatic transport have been obtained in the octupole, ${ }^{2}$ the tokamak, ${ }^{3}$ the stellarator, ${ }^{4}$ and the reversed-field pinch. 5,6 However, transport due to magnetic fluctuations has been mainly studied indirectly by measuring runaway electron flux to a limiter. $7,8,9$ Such experiments are useful for probing the magnetic fluctuations, but do not provide a local measurement of particle transport resulting from $\tilde{B}$. Magnetic transport is expected to contribute significantly to energy loss in a plasma with magnetic fluctuations. 10,11 Particle transport due to magnetic turbulence is expected to be ambipolar, 12 and recent measurements of the ambipolarity of magnetic fluctuation induced transport at the edge of a reversed-field pinch support the theoretical conclusions. ${ }^{13}$ However, the magnitude of the ambipolar flux driven by magnetic fluctuations has not been directly measured previously.

In this Letter we report on local measurements of $\left\langle\tilde{J}_{1, \theta} \tilde{B}_{r}\right\rangle$, the electron particle transport due to magnetic fluctuations in the outer region $(r / a>0.75)$ of the Madison Symmetric Torus (MST) plasma, employing an electrostatic electron energy analyzer (EEA) which incorporates a magnetic coil triplet. The outward electron particle flux just inside the last closed flux surface (LCFS) is small due to weak correlation of the fluctuations. The transported flux increases to the level of the total particle losses inside $r / a=0.85$ where integrity of the magnetic flux surfaces is believed to be destroyed.

MST 14,15 is a reversed-field pinch (RFP) toroidal confinement device. ${ }^{16}$ The level of magnetic turbulence in the RFP is higher than in the tokamak, and thus provides a suitable environment for studying magnetic transport 
phenomena. The dominant fluctuations in the RFP have been identified as resistive tearing instabilities. 17 In MST the tearing fluctuations appear in the frequency band $5-20 \mathrm{kHz}$. Magnetic fluctuation power at frequencies from 50 to $250 \mathrm{kHz}$ is due to unidentified microturbulence.

MST has a major radius of $1.5 \mathrm{~m}$ and minor radius of $0.51 \mathrm{~m}$. Probe data is obtained at $\mathrm{r} / \mathrm{a}>0.9$ in discharges of $50 \mathrm{~ms}$ duration and toroidal plasma current, $I_{\phi}=210 \mathrm{kA}$. Low current discharges $\left(I_{\phi}=120 \mathrm{kA}\right)$ with otherwise similar parameters are used to obtain transport measurements in to $r / a=0.75$. The central electron temperature is typically $100 \mathrm{eV}$, and global particle and energy confinement times are approximately $1 \mathrm{~ms}$.

The electron current is measured using a two-channel electrostatic energy analyzer $(E E A)^{18}$ with one channel aligned with the local magnetic field in each direction. A channel consists of three electrodes: an entrance aperture $0.3 \mathrm{~mm}$ in diameter that eliminates ion current (typical ion gyro-orbits have radii $\rho_{\mathrm{i}}=1 \mathrm{~cm}$ ), a cylindrical "repeller" electrode that selects collected electron energies and provides suppression of secondary electrons, and an electron current collector. The entrance electrode and the collector were maintained at machine ground potential and the repeller electrode was biased to $-50 \mathrm{~V}$, near the equilibrium floating potential. Data presented here represents the behavior of the energetic component $(E>50 \mathrm{eV})$ of the distribution which carries most or all of the parallel current at the edge of the RFP, and has an effective temperature comparable to the central electron temperature. ${ }^{18,19}$ The two channels are offset by $0.8 \mathrm{~cm}$ perpendicular to the field (toroidally). Field sensing coils are located between the two EEA channels. All five measurements $\left(J_{+}, J_{-}, B_{r}, B_{\theta}, B_{\phi}\right)$ are made at the same radial position. Records used in statistical ensembles are taken at a time just after peak plasma current, and sampled at $500 \mathrm{kHz}$. More than $1001 \mathrm{~ms}$ records are used in each ensemble average taken from 25-50 nominally identical 
discharges. Typical electron current and radial sense coil signals for the times of interest are shown in Fig. 1. The rms fluctuation amplitude in the electron current is close to $100 \%$ compared to $2 \%$ rms magnetic fluctuations. The extremely localized nature of the electron current measurement makes it sensitive to fluctuations at small spatial scales and the high fluctuation amplitude indicates the filamentary character of the electron current.

The frequency spectrum of electron current fluctuations is featureless and falls off more slowly than the $\tilde{B}$ spectrum at high frequency (Fig. 2), as expected from $\tilde{\boldsymbol{J}}=\boldsymbol{i} \mathbf{k} \times \tilde{\boldsymbol{B}}$ if $\boldsymbol{k}$ increases with frequency (the $\tilde{B}_{\phi}$ and $\tilde{B}_{\theta}$ power spectra fall off with a power law dependence as $f-5 / 2$ at high frequency, while the $\tilde{B}_{r}$ spectrum is closer to the $f-3 / 2$ prediction from MHD turbulence theory $\left.{ }^{20}\right)$. The feature in the magnetic frequency spectra at low frequencies $(f<10 \mathrm{kHz})$ is due to current fluctuations resonant at low order mode rational surfaces in the plasma core (i.e. tearing modes), ${ }^{21}$ while the fluctuation power in the electron current is dominated by local, short wavelength turbulence even at low frequency.

Fig. 3 shows the coherence and relative phase between electron current fluctuations and radial magnetic field fluctuations just inside the LCFS $(r / a=0.97)$ for $I_{\phi}=210 \mathrm{kA}$. Coherence is the absolute value of the cross-power normalized to the product of the fluctuation amplitudes,

$$
\gamma_{a b}=\frac{\left|\left\langle\tilde{a}^{*} \tilde{b}\right\rangle\right|}{\sqrt{\left\langle\tilde{a}^{2}\right\rangle} \sqrt{\left\langle\tilde{b}^{2}\right\rangle}},
$$

and the relative phase is,

$$
\phi_{a b}=\arctan \frac{\operatorname{Im}\left\langle\tilde{a}^{*} \tilde{b}\right\rangle}{\operatorname{Re}\left\langle\tilde{a}^{*} \tilde{b}\right\rangle},
$$

where the flux surface average for propagating fluctuations, $\langle\tilde{a} \tilde{b}\rangle$, is realized by making an ensemble average over many time records. The relation between the 
coherence, phase, and fluctuation amplitudes to the transported flux of electrons is, from Eqs. $1-3$,

$$
\Gamma_{r, \theta}=\frac{1}{e B} \sum_{\omega}\left|\tilde{J}_{\theta}(\omega) \| \tilde{B}_{r}(\omega)\right| \gamma_{\lrcorner B}(\omega) \cos \left(\phi_{\lrcorner B}(\omega)\right) .
$$

The coherence is $20 \%$ and the phase varies smoothly from zero to $\pi / 2$ radians across the low frequency band where the fluctuation power is concentrated. The resulting radial particle flux is $\Gamma_{\mathrm{r}}<5 \times 10^{20} \mathrm{~m}^{-2} \mathrm{~s}^{-1}$. Estimates of the total radial electron particle flux from $\mathrm{H}_{\alpha}$ emission profiles are $\Gamma_{\mathrm{r}} \approx 5 \times 10^{21} \mathrm{~m}^{-2} \mathrm{~s}^{-1}$. Magnetic fluctuation-induced electron transport is thus a negligible $\left(\Gamma_{e, \text { mag }}<0.1 \Gamma_{e, t o t a l}\right)$ channel for particle loss at the edge of the plasma. The ambipolarity of magnetic transport previously measured in MST ${ }^{13}$ coupled with the results presented in this Letter imply that the ion particle transport due to magnetic fluctuations is also negligible near the LCFS. Local electron current fluctuations at the edge, while large, are not coherent with the low frequency magnetic fluctuations resonant in the core, and the small transport resulting from the magnetic fluctuations suggests the presence of intact flux surfaces at the edge of the RFP. These results hold under the assumption that the electron current fluctuations are dominated by the fast electron current fluctuations. The caveat is not restrictive considering the observation that most of the edge current density in the RFP is carried by fast electrons. ${ }^{18,19}$ The electrostatic transport of particles at $r / a=.97$ is larger than the edge magnetic particle transport by an order of magnitude, and is comparable to the total expected particle. ${ }^{5}$

Fig. 4 shows that magnetic fluctuation induced electron transport increases with distance into the plasma. Measurements at deep insertion were made in low current $\left(I_{\phi}=120 \mathrm{kA}\right)$ discharges. The transported flux reaches levels of the order of the total expected particle flux, while measured electrostatic particle transport falls off in this region. The increase in magnetic particle 
transport is due to larger fluctuation amplitudes, rather than a rise in coherence or change in the relative phase between $\tilde{J}_{\theta}$ and $\tilde{B}_{r}$. Expected total particle flux is obtained using a transport code and is consistent with the measured density profile and edge neutral pressure. The uncertainty in the total flux is primarily due to the uncertainty in the edge pressure. Measurements of magnetic particle transport at the edge (circles in Fig. 4) were made in $I_{\phi}=210 \mathrm{kA}$ discharges and have been normalized.

Our measurements indicate that magnetic fluctuation induced transport of fast electrons can account for the expected total electron flux inside $r / a=0.85$. The fast electron diffusion coefficient, obtained by dividing $\Gamma_{\theta \text {,tast }}$ into $\nabla n_{\theta \text {,ast }}$, is $D_{\text {fast }} \approx 500 \mathrm{~m}^{2} / \mathrm{s}$. If electron transport were unconstrained by ambipolar considerations, collisionless diffusion in a region of field stochasticity would proceed at a rate given by, ${ }^{11}$

$$
D=\frac{\left\langle\tilde{B}_{r}^{2}\right\rangle}{B_{0}^{2}} v_{a v} L_{a c}
$$

where $v_{a v}$ is the average fast electron parallel velocity and $L_{a c}$ is the parallel autocorrelation length for the magnetic fluctuations. Estimates of $L_{a c}$ from magnetic wavenumber spectra agree with direct measurements of $L_{a c}$ in the scrape-off layer, giving $L_{a c}=1 \mathrm{~m}$ (the fast electrons are collisionless since their mean free path is of order $10 \mathrm{~m}$ ). For the discharge conditions discussed here and $T_{e}$,fast $=100 \mathrm{eV}$, Eq. 5 predicts $D=2000 \mathrm{~m}^{2} / \mathrm{s}$, four times the observed fast electron diffusion rate of $500 \mathrm{~m}^{2} / \mathrm{s}$.

If the stochastic diffusion mechanism described by Eq. 5 is active, a radial ambipolar electric field is established by the rapid transport of highly mobile electrons. The ambipolar field then inhibits further electron transport. The magnitude of the ambipolar electric field required to suppress electron transport in a stochastic field is given by, 22 


$$
E_{A}=-\frac{T_{\theta}}{e}\left(\frac{\nabla n}{n}+\frac{\nabla T_{\theta}}{2 T_{\theta}}\right) .
$$

For the plasma conditions of interest in this Letter, $E_{A}$ is approximately $500 \mathrm{~V} / \mathrm{m}_{\mathrm{i}}$. This number is in qualitative agreement with the measured toroidal rotation speed $\left(10^{6} \mathrm{~cm} / \mathrm{s}\right)$ of $\mathrm{C}^{+4}$ impurity ions ${ }^{23}$ as well as the toroidal propagation speed of the tearing modes ${ }^{24}$ assuming the plasma rotation is dominated by the $E \times B$ drift. In the presence of a radial ambipolar electric field, stochastic diffusion proceeds at the rate given by Eq. 5, using the ion thermal velocity, and is $50 \mathrm{~m}^{2} / \mathrm{s}$ for the conditions of interest. This value for the diffusion coefficient is consistent with estimates of $D$ from global particle balance inferred from $\mathrm{H}_{\alpha}$ emission and equilibrium plasma density profiles. The fast electron diffusion coefficient of 500 $\mathrm{m}^{2} / \mathrm{s}$ is explained by the fact that the fast electron density is roughly $10 \%$ of the bulk plasma density. Bulk electron transport is entirely suppressed by the ambipolar field.

Information about the energy dependence of magnetic particle transport is obtained by changing the bias potential on the repeller electrode of the EEA. A comparison of the transported flux and parallel electron current for three repeller bias cases is shown in Fig. 5. We see that the energy distribution of the radial flux of electrons is broader than the parallel electron distribution consistent with the expectation that higher energy electrons are preferentially transported by magnetic fluctuations (Eq.5).

In summary, we have measured for the first time the local electron particle flux produced by magnetic fluctuations. Measurements in MST show that magnetic fluctuation induced particle transport dominates the core of the RFP plasma. Numerical simulations of the RFP plasma indicate that magnetic flux surfaces are destroyed in the core ${ }^{17}$ consistent with our observation of large and increasing radial particle flux due to magnetic transport as the core is 
approached. Additionally, electron transport is found to be dominated by the high energy component of the distribution. Our results are relevant to the issue of particle transport in the core of a tokamak. Large magnetic particle transport is to be expected near rational surfaces where magnetic islands or regions of field stochasticity develop. Transport should proceed at the ion stochastic diffusion rate, with the tail of the electron distribution dominating the electron particle transport.

The authors are grateful for physics discussions with $\mathrm{Dr}$. W. Shen and the members of the MST group. This work was supported by the U.S. Department of Energy.

\footnotetext{
${ }^{1}$ P.C. Liewer, Nucl. Fusion 25, 543 (1985).

2 J.A. Schmidt, Phys. Rev. Lett. 24, 721 (1970).

${ }^{3}$ W.L. Rowan et al., Nucl. Fusion 27, 1105 (1987).

${ }^{4}$ T. Uckan et al., J. Nucl. Mater. 176-177, 693 (1990).

5 T.D. Rempel et al., Phys. Rev. Lett. 67, 1438 (1991).

${ }^{6}$ H. Ji et al., Phys. Rev. Lett. 67, 62 (1991).

${ }^{7}$ C.W. Barnes and J.D. Strachan, Nucl. Fusion 22, 1090 (1982)

${ }^{8}$ C.W. Barnes and J.D. Strachan, Phys. Fluids 26, 2668 (1983).

9 P.J. Catto et al., Phys. Fluids B 3, 2038 (1991).

10 J.D. Callen, Phys. Rev. Lett. 39, 1540 (1977).

11 A.B. Rechester and M.N. Rosenbluth, Phys. Rev Lett. 40, 38 (1978).

12 R.E. Waltz, Phys. Fluids 25, 1269 (1982).

13 W. Shen et al., Phys. Rev. Lett. 68, 1319 (1992).

14 R.N. Dexter et al., Fusion Tech. 19, 131 (1991).

15 S.C. Prager et al., Phys. Fluids B 2, 1367 (1990).

16 H.A.B. Bodin and A.A. Newton, Nucl. Fusion 19, 1255 (1980).
} 
${ }^{17}$ D.D. Schnack, E.J. Caramana, and R.A. Nebel, Phys. Fluids 28, 321 (1985).

18 J.C. Ingraham et al., Phys. Fluids B 2, 143 (1990).

${ }^{19}$ M. Stoncking et al., Bull. Am. Phys. Soc. 37, 1606 (1992), abstract only.

20 R.H. Kraichnan, Phys. Fluids 8, 1385 (1965).

${ }^{21}$ G. Chartas and S. Hokin, Phys. Fluids B 4, 4019 (1992).

22 R.W. Harvey, et al., Phys. Rev. Lett. 47, 102 (1981).

${ }^{23}$ D.J. Den Hartog, et al., Bull. Am. Phys. Soc. 38, 1979 (1993), abstract only.

${ }^{24}$ A.F. Almagri, et al., Phys. Fluids B 4, 4080 (1992). 
Figure Captions

Figure 1 Typical signals from the EEA during times of interest for discharges with $I_{\phi}=210 \mathrm{kA}$ : (a) fast electron current, (b) radial sense coil signal $\left(\dot{B}_{r}\right)$.

Figure 2 The frequency (power) spectra of electron current and magnetic field fluctuations.

Figure 3 (a) The coherence spectrum for electron current and radial magnetic field fluctuations just inside the LCFS. The dashed line indicates the confidence level. (b) The relative phase of the electron current and radial field fluctuations.

Figure 4 The radial profiles of transported electron flux due to magnetic fluctuations (squares), the electrostatic particle flux (X), and the range of expected total particle flux (dashed lines) for $I_{\phi}=120 \mathrm{kA}$. Edge measurements of magnetic transport of electrons (circles) at $I_{\phi}=210 \mathrm{kA}$ have been normalized to $I_{\phi}=120 \mathrm{kA}$.

Figure 5 The dependence of the parallel electron current and the radial electron flux due to magnetic fluctuations on the repeller electrode bias. Electrons are collected with energy exceeding the repeller bias voltage. 


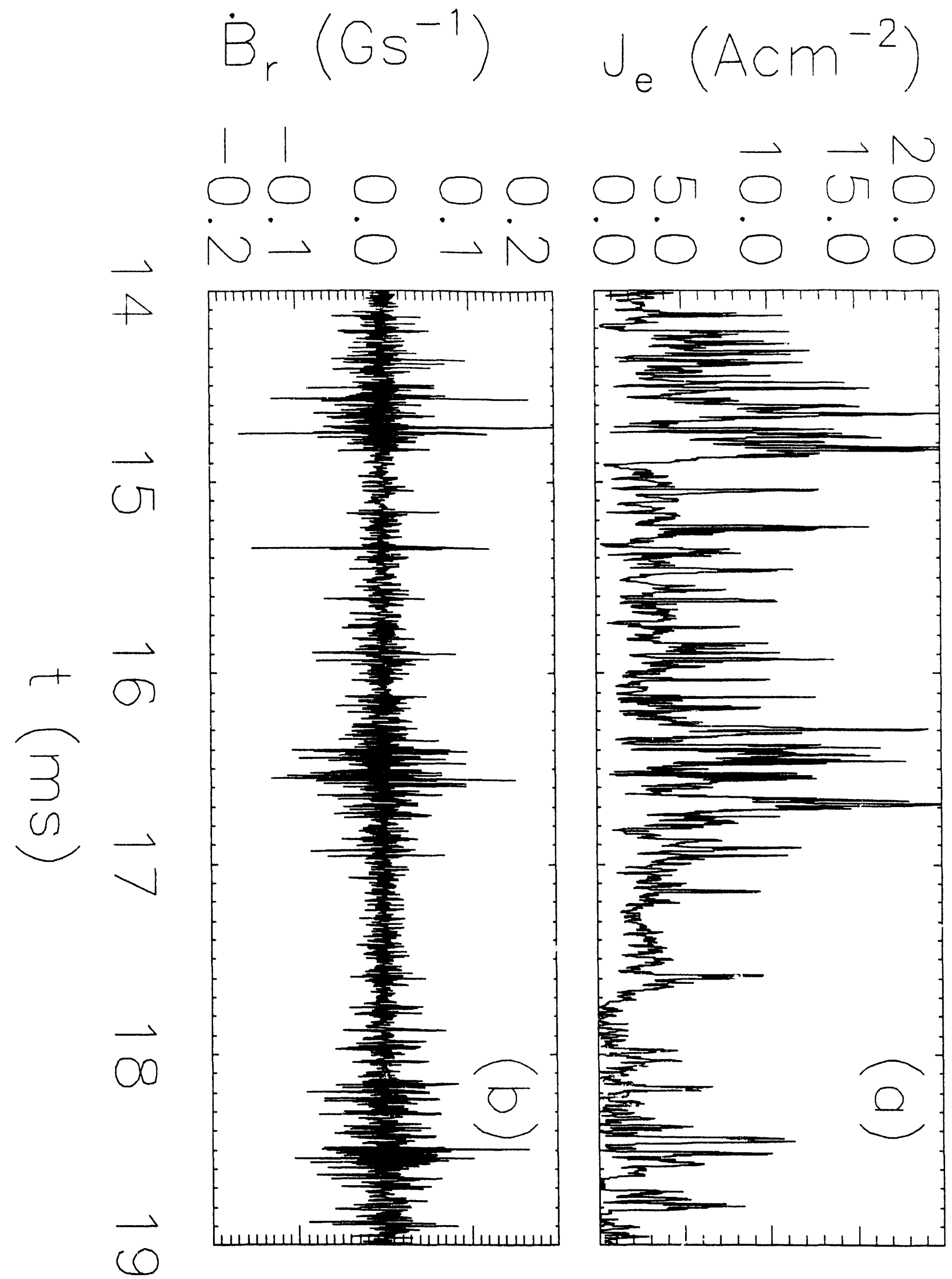




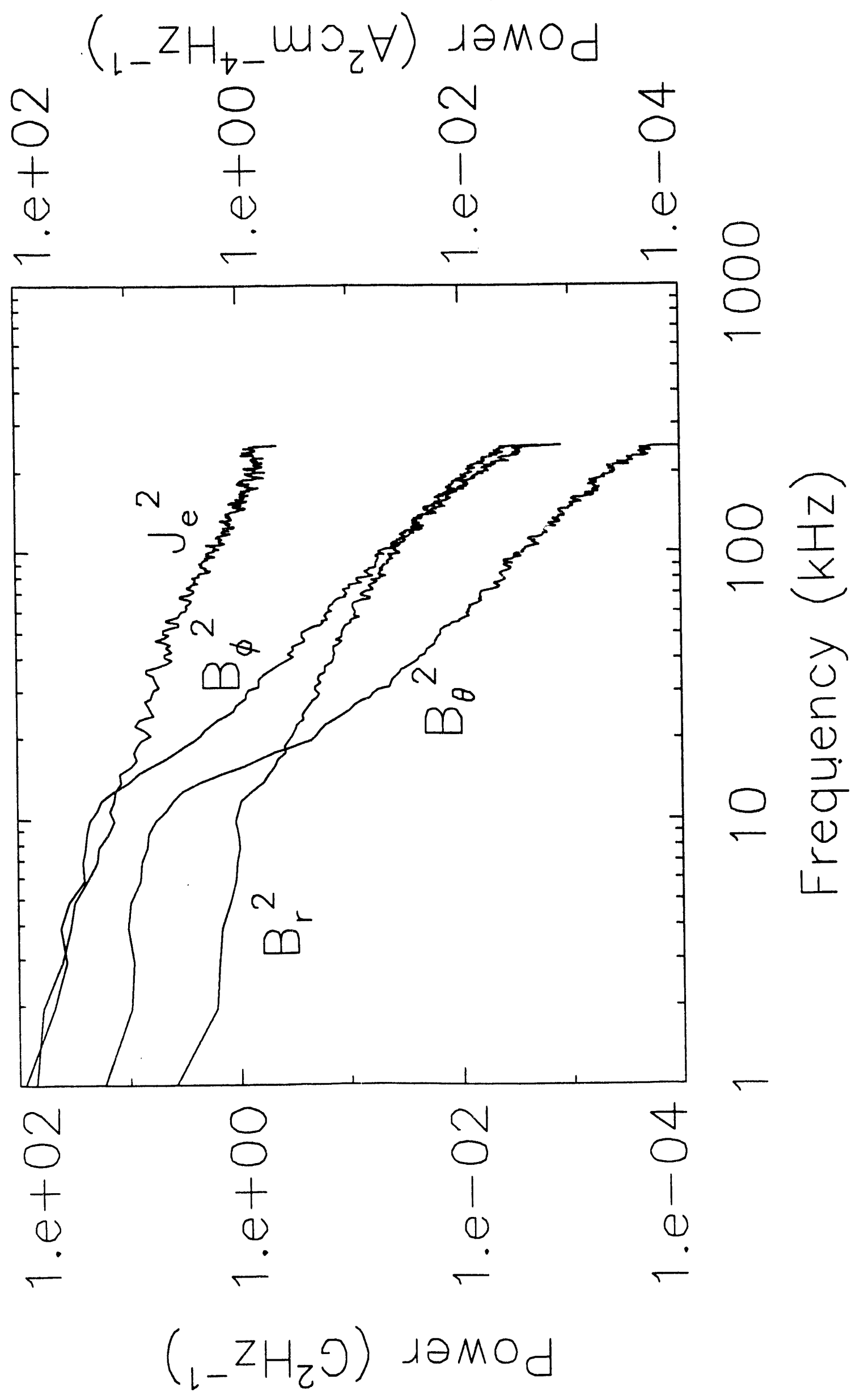




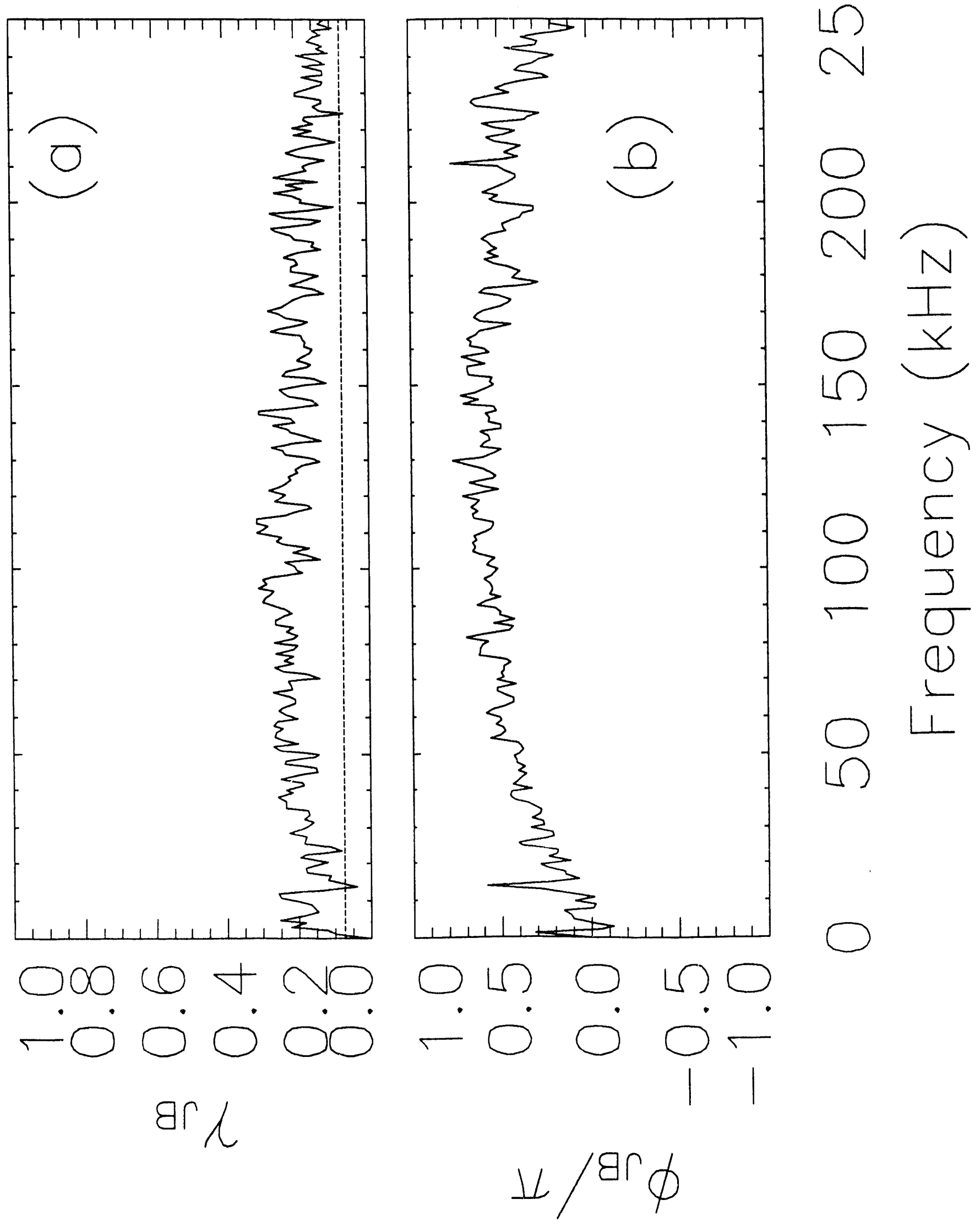




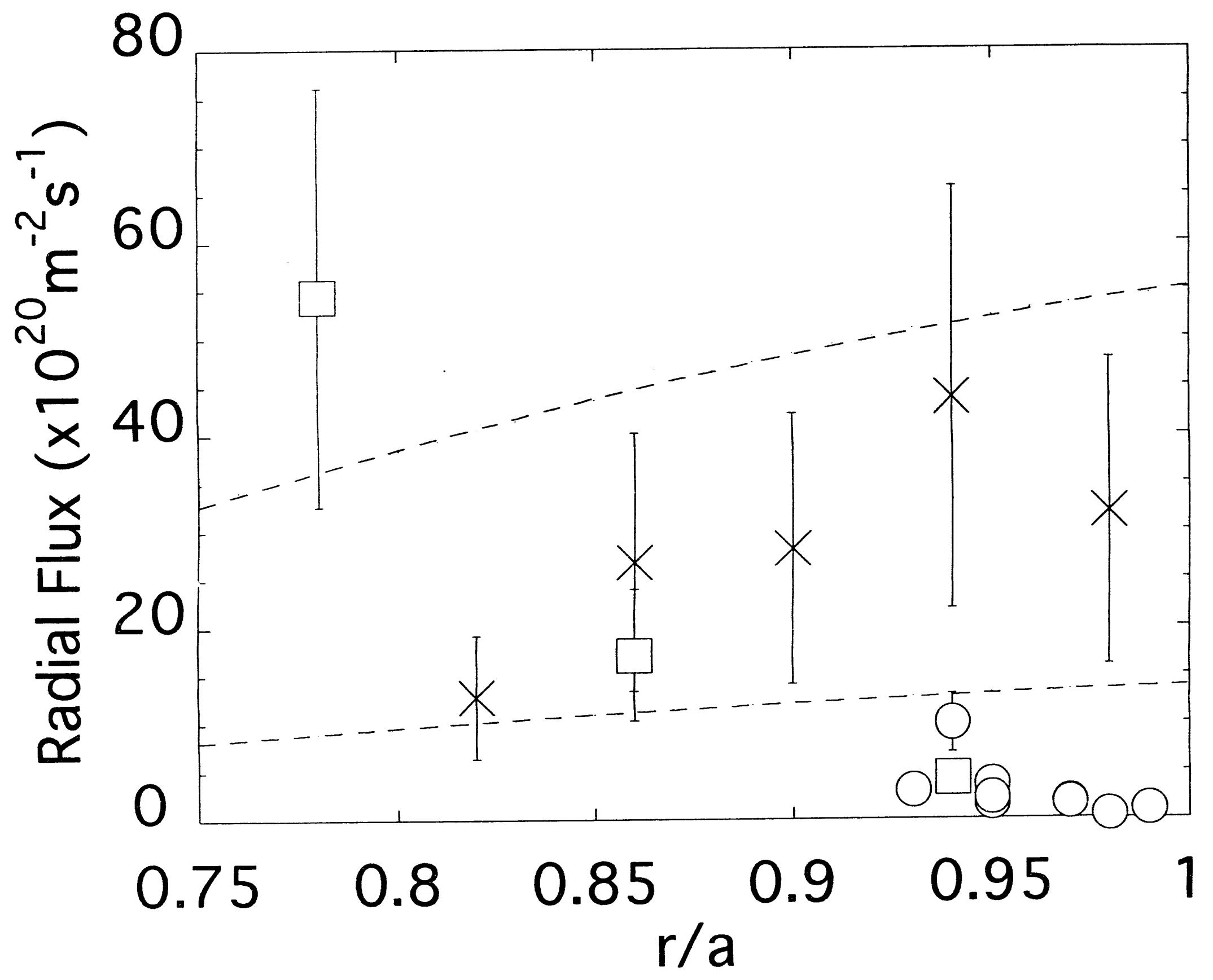

Soje 1 


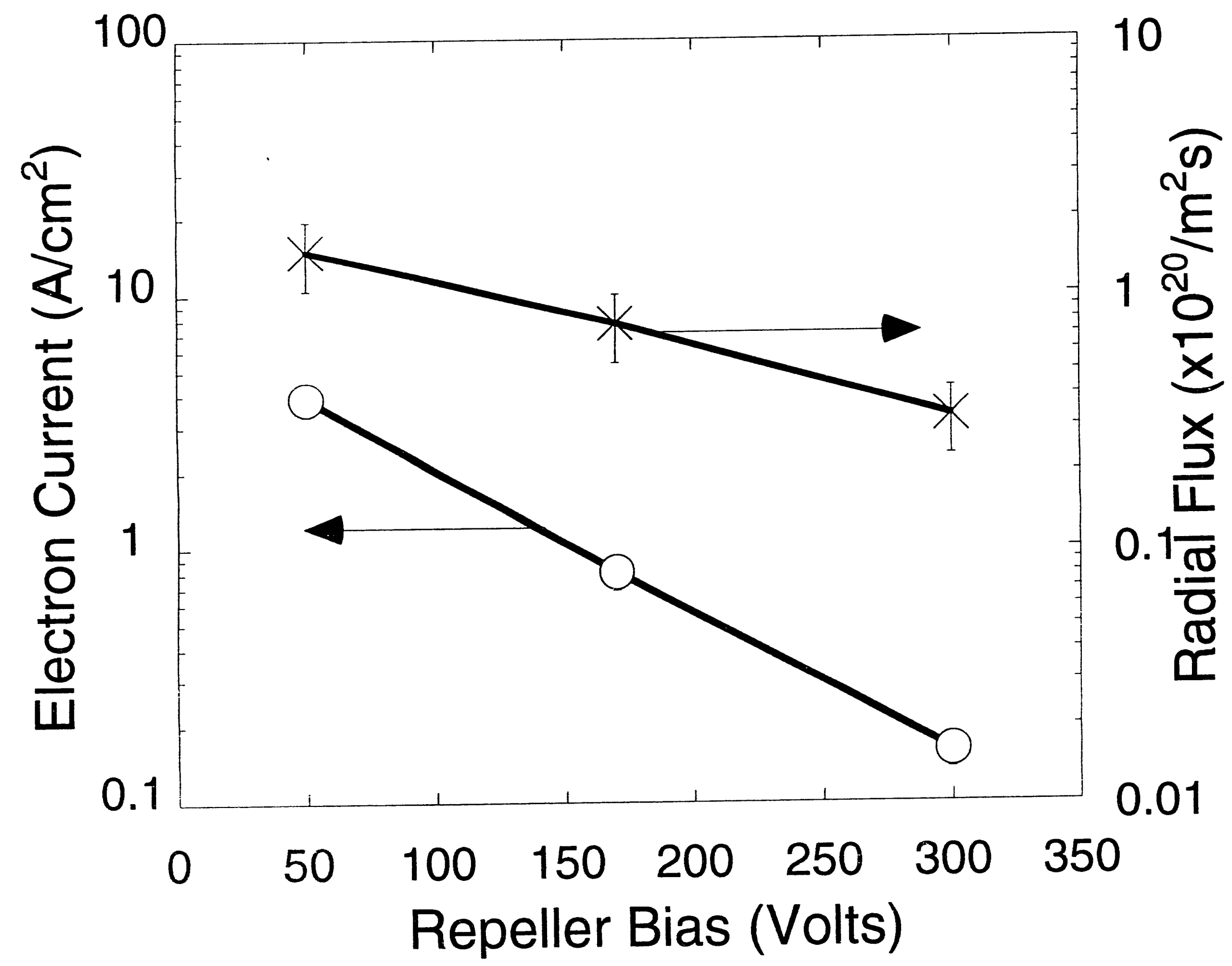




\section{EXTERNAL DISTRIBUTION IN ADDITION TO UC-20}

S.N. Rasband, Brigham Young University

T. Dolan, EG\&G Idaho, Inc.

R.A. Moyer, General Atomics

J.B. Taylor, Institute for Fusion Studies, The University of Texas at Austin

E. Uchimoto, University of Montana

F.W. Perkins, PPPL

O. Ishihara, Texas Technical University

M.A. Abdou, University of California, Los Angeles

R.W. Conn, University of California, Los Angeles

P.E. Vandenplas, Association Euratom-Etat Belge, Belgium

Centro Brasileiro de Pesquisas Firicas, Brazil

P. Sakanaka, Institute de Fisica-Unicamp, Brazil

Mme. Monique Bex, GANIL, France

J. Radet, CEN/CADARACHE, France

University of Ioannina, Greece

S. Ortolani, Istituto Gas Ionizzati, EURATON-ENEA-CNR Association, Italy

R. Andreani, Associazione EURATOM-ENEA sulla Fusione, Italy

Plasma section, Energy Fundamentals Division Electrotechnical Laboratory, Japan

Y. Kondoh, Gunma University, Kiryu, Gunma, Japan

H. Toyama, University of Tokyo, Japan

Z. Ypsjoda. University of Tokyo, Japan

FOM-Instituut voor Plasmafysica "Rijnhuizen," The Netherlands

Z. Ning, Academia Sinica, Peoples Republic of China

P. Yang, Shandong University, Peoples Republic of China

S. Zhu, University of Science \& Technology of China, People's Republic of China

I.N. Bogatu, Institute of Atomic Physics, Romania

M.J. Alport, University of Natal, Durban, South Africa

R. Storer, The Flinders University of South Australia, South Australia

B. Lehnert, Royal Institute of Technology, Sweden

Librarian, CRPP, Ecole Polytechnique Federale de Lausanne, Switzerland

B. Alper, Culham Laboratory, UK

A. Newton, UK

2 for Chicago Operations Office

5 for individuals in Washington Offices

INTERNAL DISTRIBUTION IN ADDITION TO UC-20

80 for local group and file 

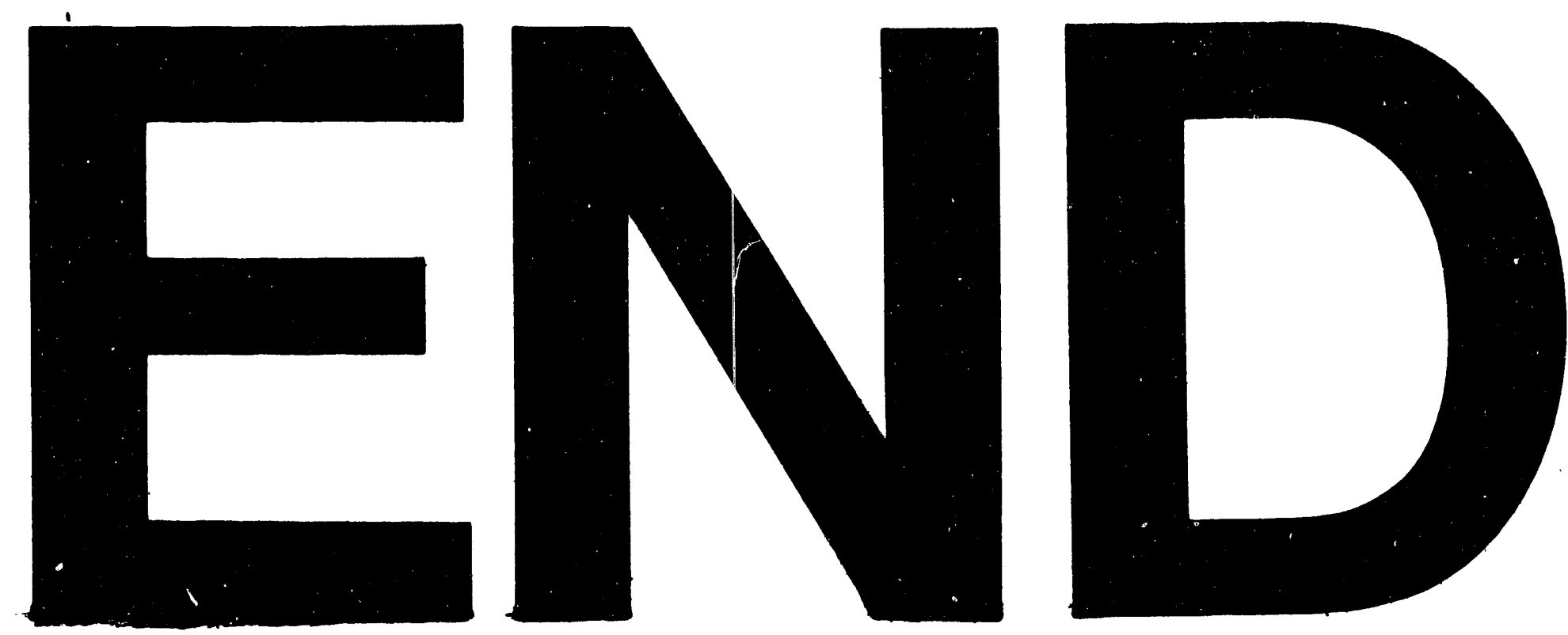

$S_{3}$
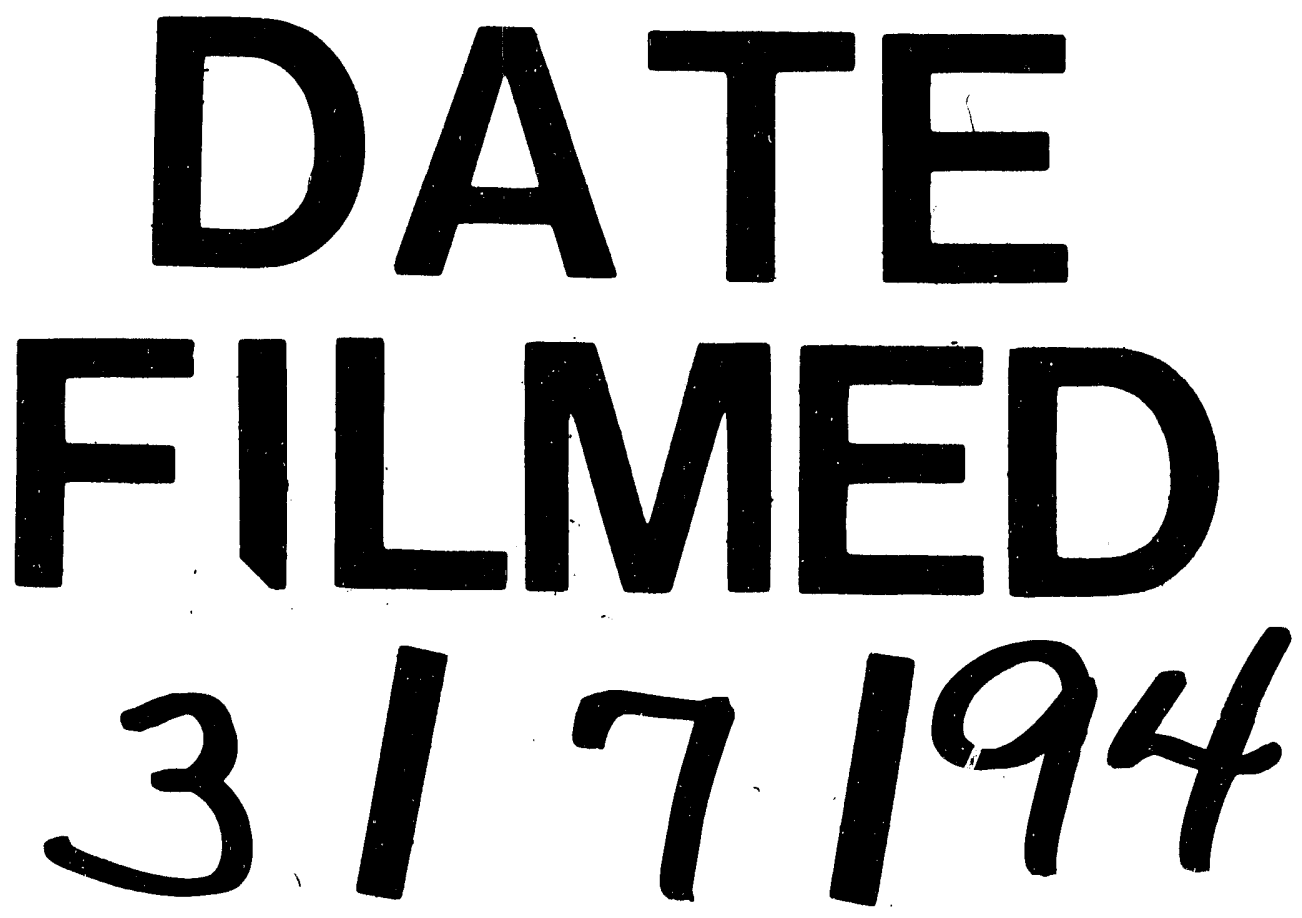

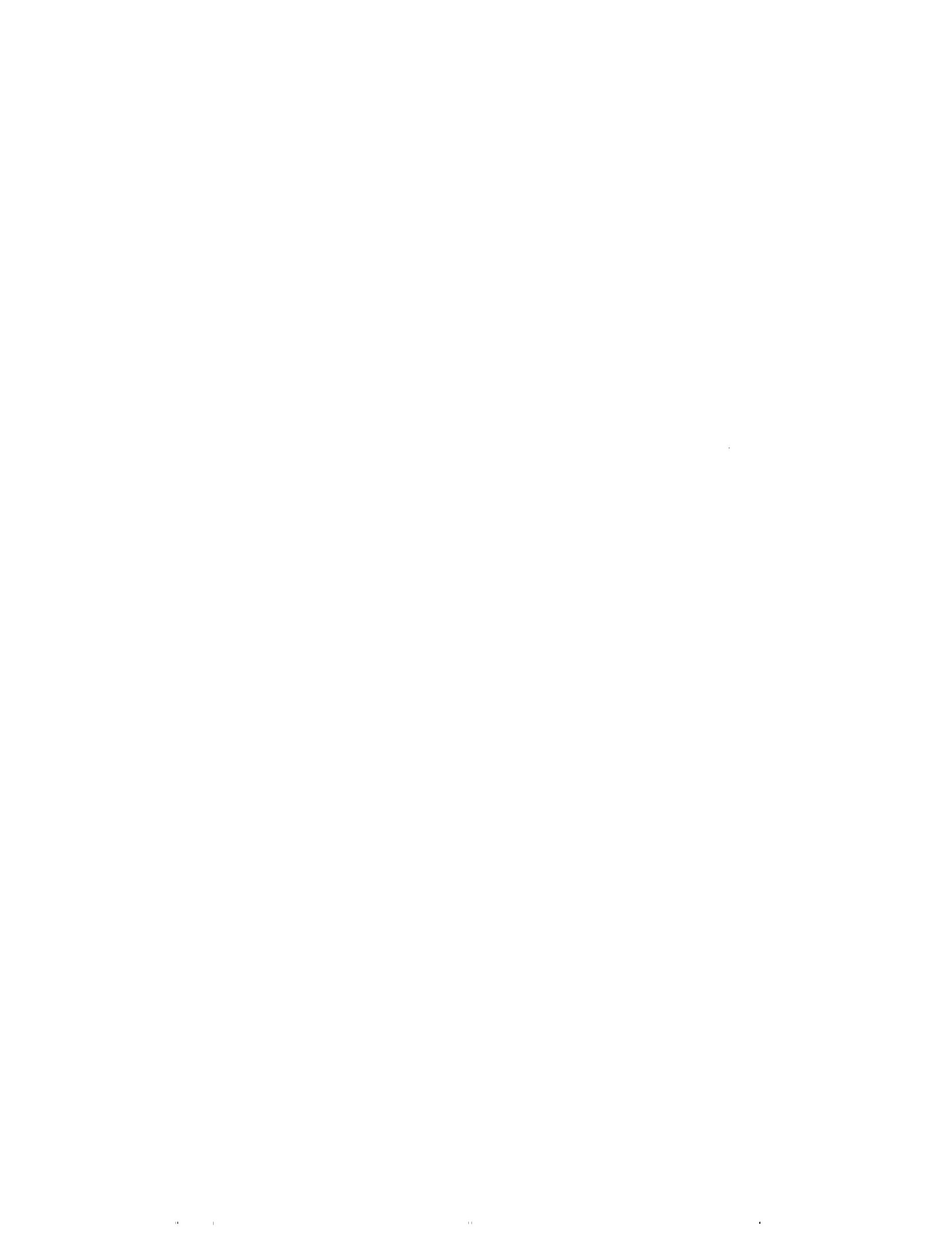